\title{
MODELO DE EMISSÃO DE POLUENTES DO TRANSPORTE RODOVIÁRIO INTERMUNICIPAL DE PASSAGEIROS NO ESTADO DE GOIÁS
}

\section{MODEL EMISSION OF POLLUTANTS OF ROAD TRANSPORT OF PASSENGERS INTER-COUNTY IN THE STATE OF GOIÁS}

\author{
Weeberb João Réquia Júnior \\ Universidade de Brasília
}

\begin{abstract}
RESUMO
Os meios de transporte são considerados os maiores responsáveis pela emissão de gases e partículas na atmosfera. A concentração elevada de poluentes no ar impacta, por exemplo, a saúde das pessoas. Nesse sentido, este trabalho teve o objetivo de estruturar um modelo de emissão de poluentes atmosféricos do transporte rodoviário intermunicipal de passageiros no estado de Goiás. Metodologicamente, foi realizado um inventário das emissões dos ônibus que compõem a frota intermunicipal do estado e em seguida gerou-se um modelo de regressão linear. Os resultados mostraram que a quantidade de passageiros transportados e a quilometragem percorrida pelos ônibus são as variáveis mais representativas no modelo gerado. Este estudo pode auxiliar a tomada de decisões para os setores de transporte, ambiental, econômico e de saúde do estado de Goiás.
\end{abstract}

Palavras-chave: Meios de transporte, Poluição atmosférica, Modelagem.

\begin{abstract}
The transport systems are the major responsible for the emission of gases and particulates in the atmosphere. The high concentration of pollutants in the air impact, for example, the people's health. Accordingly, this study aimed to design a model of air pollutants from road intercity passenger in the state of Goiás. Methodologically, was realized a inventory of emissions from buses in the fleet of intercity state and then generated into a linear regression model. The results showed that the number of passengers carried and the mileage traveled by bus are the most representative variables in the model generated. This study may help decision-making for the transport, environment, economic and health of the state of Goiás.
\end{abstract}

Keywords: Transport, Air pollution, Modeling.

\section{INTRODUÇÃO}

A necessidade do transporte de mercadorias e de passageiros está em crescimento juntamente com as cidades. A consequência desse crescimento é o aumento do tráfego de veículos nos centros urbanos e nas rodovias. Consequentemente, um maior número de carros, ônibus, caminhões, 
motocicletas gera impactos à sociedade, sobretudo, impactos ambientais e econômicos (KAMINSKI, 2001; GILBERT et al., 2003; WESTERDAHL et al., 2008; PARK et al., 2012).

Os meios de transporte respondem pela maior parte da concentração de poluentes na atmosfera (OLIVIÉ et al., 2012; WILLIAMS et al., 2012; WAKED; AFIF, 2012). A concentração elevada de partículas e gases impacta o solo (DRIVAS et al., 2011; SRIVASTAVA; KUMAR, 2012), a água (KANG; KIM, 2012; MARTELLINI et al., 2012; SHON et al., 2012), a vegetação (PANT; TRIPATHI, 2012; SANTOS et al., 2012; SAYEGH, 2012), devido à circulação de matéria pelos ciclos biogeoquímicos (MAHOWALD, 2011).

Quanto aos impactos econômicos ligados ao transporte, vê-se que o aumento da frota de veículo demanda estrutura em uma cidade e entre cidades. A instalação e a manutenção dessas estruturas ainda têm custos elevados. No período de 1995 a 2002 o Ministério dos Transportes investiu cerca de 8,6 bilhões de dólares na infra-estrutura rodoviária brasileira, buscando melhorar a condição de ir e vir da população. Por outro lado, os meios de transporte causam uma reação em cadeia que chega a impactar a economia. $O$ aumento do número de veículos gera o aumento da emissão de poluentes, que gera o aumento das doenças respiratórias, e que decorre em prejuízos ao sistema de saúde (BRAJER et al., 2012; DAVIS, 2012). Por exemplo, em São Paulo, caso o Metrô parasse, aumentaria $75 \%$ os óbitos por doenças respiratórias, o que geraria um prejuízo anual de 18 bilhões de dólares (SILVA et al., 2012).

Os estudos que buscam investigar a relação entre meios de transporte, demanda por transporte, e poluição atmosférica podem auxiliar os órgãos públicos de transporte, de meio ambiente e de saúde a implantarem medidas mais sustentáveis (CAMARGO et al., 2011; PHALEN, 2012; NING et al., 2012; TRONCOSO et al., 2012). Como exemplo dessa relação, especifica-se a importância e necessidade atual do transporte de passageiros entre as cidades Assim, este trabalho teve o objetivo de criar um modelo representativo da emissão de poluentes atmosféricos procedentes dos ônibus intermunicipais do estado de Goiás (GO). 


\section{MATERIAL E MÉTODOS}

O estudo foi realizado com base nos dados da Agência reguladora do estado de GO (AGR). As informações da AGR referem-se ao ano de 2010. Inicialmente, com os dados da frota circulante dos ônibus intermunicipais de transporte de passageiros e a quilometragem percorrida pelos ônibus, calculouse a taxa de emissão de poluentes atmosféricos por cada linha de ônibus. Esse cálculo foi realizado com base na Equação 1, conforme definição do Ministério do Meio Ambiente (MMA, 2011). O método definido pelo MMA, chamado por Top Down, já foi utilizado em outros estudos, como os de Nagpure e Gurjar, 2012, Ueda e Tomaz, 2011.

$\mathrm{E}_{\mathrm{i}, \mathrm{y}}=\frac{\mathrm{Fr}_{y} \times \mathrm{Iu}_{y} \times \mathrm{Fe}_{\mathrm{i}}}{1.000 .000}$

Onde:

$\mathrm{E}=$ taxa de emissão (toneladas $-\mathrm{t}$ );

$\mathrm{Fr}=$ frota circulante (número de veículos);

$\mathrm{lu}=$ intensidade de uso (quilometragem percorrida no ano de $2010-\mathrm{km}$ );

$\mathrm{Fe}=$ fator de emissão (grama de poluente por quilômetro rodado $-\mathrm{g} / \mathrm{km}$ );

$\mathrm{i}=$ tipo de poluente $\left(\mathrm{CO}, \mathrm{CO}_{2}, \mathrm{MP}, \mathrm{NOx}, \mathrm{NMHC}\right)$;

$y=$ linha de ônibus.

Destaca-se que para o fator de emissão (Fe) foram utilizadas as recomendações do Programa de Controle da Poluição do Ar por Veículos Automotores - PROCONVE (CONAMA, 1990).

A taxa de emissão de cada poluente calculada pela Equação 1 irá representar a variável dependente do modelo proposto por este trabalho. Quanto às variáveis independentes, passaram por duas etapas antes da geração do modelo final. 
A primeira etapa consistiu em selecionar as variáveis independentes que representam melhor a emissão dos poluentes atmosféricos. Para isso, utilizouse o método de regressão linear múltipla do tipo StepWise- forward. Esse método consiste em incluir uma nova variável independente a cada passo de elaboração do modelo. Foram consideradas como variáveis independentes a extensão de cada linha (EL), o tamanho da frota de ônibus (TF), a receita das empresas que operam as linhas (RE), o total de viagens realizadas (TV), o total de passageiros transportados (PT) e o total de quilômetros percorridos (TQ).

A segunda etapa referiu-se na geração do modelo final. Com a seleção das variáveis mais significativas na etapa 1 , gerou-se o modelo de emissão de poluentes do transporte rodoviário intermunicipal de passageiros no estado de GO (MEPTRIP), representado pela Equação 2.

$\operatorname{MEPTRIP}_{\beta}=a+b_{1} \cdot x_{1}+b_{2} \cdot x_{2}+\ldots+b_{1} \cdot x_{1}$

Equação (2)

Onde:

$\beta=$ poluente considerado no modelo (variável dependente);

$a=$ constante de regressão;

$b_{1}, b_{2}, \ldots, b_{i}=$ coeficiente das variáveis incluídas no modelo;

$\mathrm{x}_{1}, \mathrm{x}_{2}, \ldots, \mathrm{x}_{\mathrm{i}}=$ variável independente.

Por fim, foi utilizado o software BioEstat 5.0 como ferramenta estatística para o desenvolvimento da presente pesquisa.

\section{RESULTADOS E DISCUSSÃO}

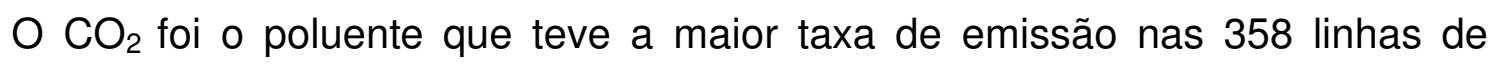
ônibus intermunicipais do estado de GO, uma média de 295,70 t no ano de 2010. O MP foi o poluente com a menor média de taxa de emissão, 0,10 t. Todos os poluentes tiveram um desvio padrão maior que a média, o que 
representa um coeficiente de variação alto (Tabela 1). Esses valores elevados do coeficiente de variação dos poluentes podem estar associados ao alto valor de variação das variáveis estudadas (Tabela 2).

Tabela 1 - Valores descritivos das emissões de poluentes $(t)$ das linhas intermunicipais do estado de GO do ano de 2010

\begin{tabular}{c|c|c|c}
\hline POLUENTE & MÉDIA & DP & MÁXIMO \\
\hline $\mathrm{CO}$ & 0,52 & 2,13 & 37,33 \\
\hline $\mathrm{CO}_{2}$ & 295,70 & 1220,69 & 21341,84 \\
\hline $\mathrm{MP}$ & 0,10 & 0,42 & 7,33 \\
\hline $\mathrm{NO}{ }_{\mathrm{x}}$ & 0,16 & 0,65 & 11,33 \\
\hline $\mathrm{NMHC}$ & 2,57 & 10,62 & 185,69 \\
\hline
\end{tabular}

DP - desvio padrão

Em outros estudos foram encontradas também a predominância da emissão de $\mathrm{CO}_{2}$ pelo setor de transporte (SHINDELL et al., 2012; RAO et al., 2012; KHEDAIRIA; KHADIR, 2012; BRONDFIELD et al., 2012). Contudo, isso não elimina o impacto das emissões, mesmo que reduzidas, de outros poluentes. Pesquisas já apontaram que as emissões do setor de transporte são suficientes para afetar a saúde das pessoas (BERNSTEIN et al., 2004; CAMARGO et al., 2011; TOLEDO; NARDOCCI, 2011).

No ano de 2010 foram transportados mais de 12 milhões de passageiros entre os municípios do estado de GO, em um total de 558 mil viagens. O 492 ônibus intermunicipais que compõem a frota do estado percorreram mais de 52 milhões de quilômetros. A extensão de todas as linhas percorridas pelos ônibus intermunicipais é entorno de 50 mil quilômetros. Percebe-se que não há um padrão de observações para as variáveis TQ, EL, TV, TF, RE e TP entre as 358 linhas do estado de GO, devido o valor de desvio padrão ser maior que a média. (Tabela 2).

Tabela 2 - Valores descritivos das variáveis independentes utilizadas no modelo 


\begin{tabular}{c|c|c|c|c|c}
\hline $\begin{array}{c}\text { VARIÁVE } \\
\text { L }\end{array}$ & MÉDIA & DP & MÁXIMO & MíNIMO & SOMA \\
\hline TQ & $\begin{array}{c}147.808,6 \\
5\end{array}$ & $\begin{array}{c}190.467,6 \\
8\end{array}$ & $\begin{array}{c}2.421 .000,0 \\
0\end{array}$ & $\begin{array}{c}1.512,0 \\
0\end{array}$ & $\begin{array}{c}52.915 .497,0 \\
0\end{array}$ \\
\hline EL & 140,77 & 115,06 & 560,00 & 13,00 & $50.397,00$ \\
\hline TV & $1.560,58$ & $2.915,63$ & $40.350,00$ & 10,00 & $558.689,00$ \\
\hline TF & 1,37 & 0,98 & 10,00 & 0 & 492 \\
\hline RE & $255.297,2$ & $488.361,7$ & $6.163 .536,6$ & 0,00 & $91.396 .414,1$ \\
\hline PT & $54.672,50$ & $79.709,59$ & $1.186 .693,0$ & 111,00 & $12.412 .754,3$ \\
& & 1 & 0 & & 5 \\
\hline
\end{tabular}

DP - desvio padrão

Os resultados encontrados no modelo foram semelhantes para cada tipo de poluente. A diferença numérica decimal era a partir da ordem de $10^{-3}$, sobretudo, para o erro do quadrado médio (QM erro). Assim, considerou-se o resultado de maneira consolidada para todos os tipos de poluentes analisados (Tabela 3).

Tabela 3- Resultado da regressão linear múltipla do tipo StepWise - forward

\begin{tabular}{ccccccc}
$\begin{array}{c}\text { VARIÁVEIS NO } \\
\text { MODELO }\end{array}$ & $\mathbf{R}$ & $\mathbf{R}^{2}$ & $\begin{array}{c}\text { VARIAÇÃOO } \\
\mathbf{R}^{2}\end{array}$ & $\begin{array}{c}\text { P- } \\
\text { VALOR }\end{array}$ & $\begin{array}{c}\text { QM } \\
\text { ERO }\end{array}$ & $\begin{array}{c}\text { VARIÁVEL } \\
\text { INCLUÍDA }\end{array}$ \\
\hline PT & 0,8622 & $74,34 \%$ & $74,34 \%$ & 0,000 & 1,1733 & PT \\
PT, TQ & 0,9135 & $83,44 \%$ & $9,11 \%$ & 0,000 & 0,7590 & TQ \\
PT, TQ, RE & 0,914 & $83,54 \%$ & $0,09 \%$ & 0,000 & 0,7569 & RE \\
PT, TQ, RE, TV & 0,9163 & $83,95 \%$ & $0,42 \%$ & 0,000 & 0,7398 & TV \\
PT, TQ, RE, TV, TF & 0,9183 & $84,33 \%$ & $0,38 \%$ & 0,000 & 0,7244 & TF \\
PT, TQ, RE, TV, TF, & & & & & & \\
EL & 0,9203 & $84,70 \%$ & $0,36 \%$ & 0,000 & 0,7096 & EL \\
\hline
\end{tabular}

Conforme a Tabela 3, nota-se que o $p$-valor para ambas variáveis incluídas no modelo é significativo $(<0,001)$. Com isso, é possível validar a hipótese alternativa $\left(H_{1}: b \neq 0\right)$ - há uma relação de dependência. A maior variação 
ocorrida no coeficiente de variação $\left(r^{2}\right)$ foi com a inserção da variável TQ. Houve uma variação de $9,11 \%$. As demais variações foram inferiores a $0,5 \%$. Nesse sentido, pode-se concluir que o modelo representaria melhor a realidade com a adoção das seguintes variáveis independentes: passageiros transportados (PT) e total de quilômetros percorridos (TQ). Pesquisas no Canadá (GILBERT et al., 2007; SABALIAUSKAS et al., 2012) e na China (CAI et al., 2009; SONG et al., 2012; NING et al., 2012) já identificaram a influência dessas variáveis na emissão de poluentes atmosféricos.

Com a escolha das variáveis independentes mais significantes para geração do modelo, foi rodado o teste de regressão linear múltipla com a as variáveis independentes PT e TQ (Tabela 4).

Tabela 4- Modelo de regressão para os poluentes atmosféricos com base nas variáveis independentes mais significativas

\begin{tabular}{c|c|c|c|c|c}
\hline $\begin{array}{c}\text { POLUENTE } \\
(\mathbf{Y})\end{array}$ & $\begin{array}{c}\text { INTERCEPTO } \\
(\mathbf{a})\end{array}$ & $\begin{array}{c}\text { COEFICIENTE } \\
\mathbf{( b}_{\mathbf{1}} \mathbf{n}\end{array}$ & $\begin{array}{c}\text { COEFICIENTE } \\
\mathbf{( b}_{\mathbf{2}} \mathbf{p}\end{array}$ & $\begin{array}{c}\mathbf{p}- \\
\text { valor }\end{array}$ & $\mathbf{R}^{\mathbf{2}}$ \\
$\mathrm{CO}$ & $-0,7249$ & 0,0000 & 0,0000 & $<0,001$ & 0,83 \\
$\mathrm{CO}_{2}$ & -4.143 .083 & 0,0078 & 0,0030 & $<0,001$ & 0,83 \\
MP & $-0,1424$ & 0,0000 & 0,0000 & $<0,001$ & 0,84 \\
NOx & $-0,2199$ & 0,0000 & 0,0000 & $<0,001$ & 0,84 \\
NMHC & -36.048 & 0,0001 & 0,0000 & $<0,001$ & 0,85 \\
\hline
\end{tabular}

$\mathrm{b}_{1}=$ coeficiente da variável PT

$\mathrm{b}_{2}=$ coeficiente da variável TQ

As duas variáveis independentes apresentaram coeficientes betas semelhantes, ambos com significância $(p<0,01)$. $O$ coeficiente de determinação foi semelhante nos modelos gerados, com variação de 0,83 a 0,85. Lembrando-se que cada poluente representou um modelo. As maiores diferenças entre os modelos gerados foram encontradas no coeficiente do intercepto alfa. $\mathrm{O}$ modelo representado pelo $\mathrm{CO}_{2}$ foi o que apresentou o menor valor do intercepto. Contudo, é possível afirmar pelo valor do intercepto $(\neq 0)$ que as variáveis independentes utilizadas influenciam na taxa de emissão de 
poluentes (Tabela 4). Assim, o modelo de emissão de poluentes do transporte rodoviário intermunicipal de passageiros no estado de GO (MEPTRIP) pode ser representado pelas equações 3 a 7 .

$$
\begin{array}{lr}
\text { MEPTRIP }_{\mathrm{CO}}=-0,7249+\mathrm{PT}+\mathrm{TQ} & \text { Equação } 3 \\
\text { MEPTRIP }_{\mathrm{CO} 2}=-4.143 .083+(0,0078 . \mathrm{PT})+(0,0030 . T \mathrm{TQ}) & \text { Equação } 4 \\
\text { MEPTRIP }_{\mathrm{MP}}=-0,1424+\mathrm{PT}+\mathrm{TQ} & \text { Equação } 5 \\
\text { MEPTRIP }_{\mathrm{NOx}}=-0,2199+\mathrm{PT}+\mathrm{TQ} & \text { Equação } 6 \\
\text { MEPTRIP }_{\mathrm{NMHC}}=-36.048+(0,001 . \mathrm{PT})+\mathrm{TQ} & \text { Equação } 7
\end{array}
$$

No entanto, a proposta não é transportar menos passageiros, mas sim transportar mais passageiros em rotas eficientes e com veículos de baixa emissão. A gestão eficiente das 358 linhas de ônibus intermunicipais do GO que tem o objetivo de transportar uma média anual de 12 milhões de passageiros entre os municípios do estado iria influenciar na redução da emissão de poluentes. A regulação e a gestão do transporte são características que proporcionam um retorno ambiental, econômico e social (ABOU-ALI; THOMAS, 2011; PHALEN, 2012; NING et al., 2012; TRONCOSO et al., 2012).

Diante do exposto, há ainda perguntas para serem investigadas em estudos futuros. Sugere-se que as próximas pesquisas avaliem a relação de toda a matriz do transporte, ou seja, automóveis particulares, ônibus urbanos, caminhões, etc. O teste de outras variáveis também poderia ser analisado em um novo modelo.

Por fim, sugere-se 0 aperfeiçoamento na criação de modelos como continuidade da presente pesquisa. Estudos atuais estão criando modelos de regressão que tem o objetivo de avaliar a relação de poluição atmosférica com 
variáveis de uso do solo (PAULEIT; DUHME, 2000; SU et al., 2009; AMIN et al., 2012; MUKERJEE et al., 2012; LI et al., 2012). Essa técnica é conhecida como Land Use Regression - LUR. Agregaria conhecimento a melhor compreensão da relação entre transporte, poluição atmosférica e uso do solo.

\section{CONCLUSÃO}

Este trabalho teve o objetivo de avaliar a relação entre o sistema de transporte intermunicipal de passageiros do estado de GO com a poluição atmosférica. Verificou-se que a quantidade de passageiros transportados e a quilometragem percorrida por cada ônibus são as variáveis mais significativas para representar o modelo de poluição atmosférica da relação estudada.

Sabe-se da influência negativa ao meio ambiente, sobretudo, à saúde das pessoas, que a concentração elevada de gases e partículas na atmosfera provoca. Nesse sentido, o órgão público do estado de GO pode basear nos resultados deste estudo como motivação para a melhor gestão e controle das empresas que operam as linhas transporte intermunicipal de passageiros do estado.

\section{REFERÊNCIAS BIBLIOGRÁFICAS}

ABOU-ALI, H.; THOMAS, A. Regulating traffic to reduce air pollution in Greater Cairo, Egypt. ,2011.

AMIN, ARSHAD; AMIN, ARIF; SINGH, S. K. Study of urban land use dynamics in Srinagar city using geo-spatial approach. Bulletin of environmental and scientific research, v. 1, n. 2, p. 18-24, 2012.

BERNSTEIN, J. A.; ALEXIS, N.; BARNES, C.; et al. Health effects of air pollution. The Journal of allergy and clinical immunology, v. 114, n. 5, p. 1116-23, 2004.

BRAJER, V.; HALL, J.; RAHMATIAN, M. Air Pollution , Its Mortality Risk , and Economic Impacts in Tehran, Iran. Iranian Journal Publising Health, v. 41, n. 5, p. 31-38, 2012.

BRONDFIELD, M. N.; HUTYRA, L. R.; GATELY, C. K.; RACITI, S. M.; PETERSON, S. A. Modeling and validation of on-road $\mathrm{CO} 2$ emissions inventories at the urban regional scale. Environmental pollution, v. 170, p. 113-23, 2012. 
CAI, M.; YIN, Y.; XIE, M. Prediction of hourly air pollutant concentrations near urban arterials using artificial neural network approach. Transportation Research Part D: Transport and Environment, v. 14, n. 1, p. 32-41, 2009.

CAMARGO, N. V. A.; COSTA, N. R.; MIRAGLIA, S. G. E. K. Poluição atmosférica - Análise sobre as medidas de controle da poluição atmosférica e o sistema de informação do Parque do Ibirapuera. Gepros - Gestão da Produção, Operações e Sistemas, , n. 2, p. 87-100, 2011.

CONAMA. Resolução Conama número 003. (Ministério do Meio Ambiente, Ed.), 1990. Brasília.

DAVIS, M. E. Recessions and Health: The Impact of Economic Trends on Air Pollution in California. American journal of public health, p. 1-6, 2012.

DRIVAS, P.; BOWERS, T.; YAMARTINO, R. Soil mixing depth after atmospheric deposition. I. Model development and validation. Atmospheric Environment, v. 45, n. 25, p. 4133-4140, 2011.

GILBERT, N. L.; GOLDBERG, M. S.; BROOK, J. R.; JERRET, M. The influence of highway traffic on ambient nitrogen dioxide concentrations beyond the immediate vicinity of highways. Atmospheric Environment, v. 41, p. 2670-2673, 2007.

GILBERT, N. L.; WOODHOUSE, S.; STIEB, D. M.; BROOK, J. R. Ambient nitrogen dioxide and distance from a major highway. The Science of the total environment, v. 312, n. 1-3, p. 43-6, 2003.

KAMINSKI, W. Evaluation of air pollution level by means of artificial neural network - multilayer perceptron. Handbook of Conveying and Handling of Particulate Solids, p. 739-744, 2001.

KANG, J.; KIM, J. Y. Portable RF-Sensor System for the Monitoring of Air Pollution and Water Contamination. Journal of Analytical Methods in Chemistry, v. 2012, p. 1-5, 2012.

KHEDAIRIA, S.; KHADIR, M. T. Impact of clustered meteorological parameters on air pollutants concentrations in the region of Annaba, Algeria. Atmospheric Research, v. 113, p. 89-101, 2012.

LI, L.; WU, J.; WILHELM, M.; RITZ, B. Use of generalized additive models and cokriging of spatial residuals to improve land-use regression estimates of nitrogen oxides in Southern California. Atmospheric Environment, v. 55, p. 220-228, 2012.

MAHOWALD, N. Aerosol indirect effect on biogeochemical cycles and climate. Science, v. 334, n. 6057, p. 794-6, 2011.

MARTELLINI, T.; JONES, K. C.; SWEETMAN, A.; et al. The contribution of waste water treatment plants to PBDEs in ambient air. Environmental pollution, v. 169, p. 242-247, 2012.

MMA. $1^{\circ}$ Inventário nacional de emissoes atmosféricas por veículos automotores rodoviários. 1st ed. Brasília, Brasil: Ministério do Meio Ambiente, 2011.

MUKERJEE, S.; WILLIS, R. D.; WALKER, J. T.; et al. Seasonal effects in land use regression models for nitrogen dioxide, coarse particulate matter, and gaseous ammonia in Cleveland, Ohio. Atmospheric Pollution Research, v. 3, n. 3, p. 352-361, 2012. 
NAGPURE, A. S.; GURJAR, B. R. Development and evaluation of Vehicular Air Pollution Inventory model. Atmospheric Environment, v. 59, p. 160-169, 2012. Elsevier Ltd.

NING, Z.; WUBULIHAIREN, M.; YANG, F. PM, NOx and butane emissions from on-road vehicle fleets in Hong Kong and their implications on emission control policy. Atmospheric Environment, v. 61, n. 2, p. 265-274, 2012.

OLIVIÉ, D. J. L.; CARIOLLE, D.; TEYSSĖDRE, H.; et al. Modeling the climate impact of road transport, maritime shipping and aviation over the period 1860-2100 with an AOGCM. Atmospheric Chemistry and Physics, v. 12, n. 3, p. 1449-1480, 2012.

PANT, P. P.; TRIPATHI, A. K. Analysis of some biochemical parameters of plants as indicator of air pollution. Journal of environmental science, computer science and engineering \& technology, v. 1, n. 1, p. 14-21, 2012.

PARK, D.; OH, M.; YOON, Y.; PARK, E.; LEE, K. Source identification of PM10 pollution in subway passenger cabins using positive matrix factorization. Atmospheric Environment, v. 49, p. 180-185, 2012.

PAULEIT, S.; DUHME, F. Assessing the environmental performance of land cover types for urban planning. Landscape and Urban Planning, v. 52, p. 1-20, 2000.

PHALEN, R. F. Introduction to air pollution science: a publick health perspective. 1st ed. Burlington: Alpha Press, 2012.

RAO, S.; CHIRKOV, V.; DENTENER, F.; et al. Environmental modeling and methods for estimation of the global health impacts of air pollution. Environment model assessment, 2012. SABALIAUSKAS, K.; JEONG, C.-H.; YAO, X.; et al. Five-year roadside measurements of ultrafine particles in a major Canadian city. Atmospheric Environment, v. 49, p. 245-256, 2012.

SANTOS, O. M.; GUEDES, M. L. S.; ARAÚJO, C. V. M. Potencial de espécies vegetais nativas do pólo industrial de Camaçari (BA, Brasil) como acumuladoras de elementos químicos contidos na poluição atmosférica. Journal of the Brazilian Society of Ecotoxicology, v. 7, n. 1, p. 15-20, 2012.

SAYEGH, S. P. Forest biomonitoring of the largest Slovene thermal power plant with respect to reduction of air pollution. Environmental monitoring and assessment, , n. 2010, 2012.

SHINDELL, D.; KUYLENSTIERNA, J. C. I.; VIGNATI, E.; et al. Simultaneously mitigating nearterm climate change and improving human health and food security. Science, v. 335, n. 183, p. 183-9, 2012.

SHON, Z.-H.; KIM, K.-H.; SONG, S.-K.; et al. Relationship between water-soluble ions in PM2.5 and their precursor gases in Seoul megacity. Atmospheric Environment, v. 59, p. 540-550, 2012. 
SILVA, C. B. P.; SALDIVA, P. H. N.; AMATO-LOURENÇO, L. F.; RODRIGUES-SILVA, F.; MIRAGLIA, S. G. E. K. Evaluation of the air quality benefits of the subway system in São Paulo, Brazil. Journal of environmental management, v. 101, p. 191-6, 2012.

SONG, W. W.; HE, K. B.; LEI, Y. Black carbon emissions from on-road vehicles in China, 19902030. Atmospheric Environment, v. 51, p. 320-328, 2012.

SRIVASTAVA, K. P.; KUMAR, S. V. Impact of Air-Pollution on pH of soil of Saran, Bihar, India. Research Journal of Recent Sciences, v. 1, n. 4, p. 9-13, 2012.

SU, J. G.; JERRETT, M.; BECKERMAN, B. A distance-decay variable selection strategy for land use regression modeling of ambient air pollution exposures. The Science of the total environment, v. 407, n. 12, p. 3890-3898, 2009.

TOLEDO, G. I. F. M.; NARDOCCI, A. C. Poluição veicular e saúde da população : uma revisão sobre o município de São Paulo ( SP ), Brasil. Revista Brasileira Epidemiologia, v. 14, n. 3, p. 445-454, 2011.

TRONCOSO, R.; GRANGE, L. DE; CIFUENTES, L. A. Effects of environmental alerts and preemergencies on pollutant concentrations in Santiago, Chile. Atmospheric Environment, v. 61, p. 550-557, 2012.

UEDA, A. C.; TOMAZ, E. Inventário de emissão de fontes veiculares da região metropolinata de Campinas, São Paulo. Química Nova, v. 34, n. 9, p. 1496-1500, 2011.

WAKED, A.; AFIF, C. Emissions of air pollutants from road transport in Lebanon and other countries in the Middle East region. Atmospheric Environment, v. 61, p. 446-452, 2012.

WESTERDAHL, D.; FRUIN, S. A.; FINE, P. L.; SIOUTAS, C. The Los Angeles International Airport as a source of ultrafine particles and other pollutants to nearby communities. Atmospheric Environment, v. 42, p. 3143-3155, 2008.

WILliamS, J. E.; WEELE, M. VAN; VELTHOVEN, P. F. J. VAN; et al. The Impact of Uncertainties in African Biomass Burning Emission Estimates on Modeling Global Air Quality, Long Range Transport and Tropospheric Chemical Lifetimes. Atmosphere, v. 3, p. 132-163, 2012.

Recebido: 01/02/2014

Aprovado: 25/05/2014 